\title{
GESCHICHTSPHILOSOPHIE UND ZUKUNFTSETHIK
}

\author{
Johannes Rohbeck \\ Technische Universität Dresden
}

\begin{abstract}
To further develop the ethical dimension of philosophy of history, action-oriented and directed towards the future, it is worth to establish a bridge with the ethics of the future, meanwhile established itself as an independent discourse. Her questions are raised about the moral responsibility related to generations temporally near and far. Clearly, in this context time relationships play an important role - not just the relationship of past, present and future in general - but especially those times captured by the look of the next or future generations, through which one must assume at the outset responsibility.
\end{abstract}

Keywords: ethics, philosophy of history, responsibility, future generations.

Resumo: Para continuar a desenvolver a dimensão ética de uma filosofia da história orientada para a ação e direcionada para o futuro, vale a pena estabelecer uma ponte com a ética do futuro, que entrementes se firmou como um discurso independente. Nela são colocadas questões acerca da responsabilidade moral relacionada a gerações temporalmente próximas ou distantes. É evidente que neste contexto relações de tempo desempenham um papel importante - não apenas a relação do passado, presente e futuro em geral -, mas também especialmente aqueles tempos captados pelo olhar das gerações próximas ou futuras, pelas quais se deve assumir, desde já, a responsabilidade.

Palavras-chave: ética, filosofia da história, responsabilidade, futuro, gerações.

Um die ethische Dimension einer handlungsorientierten und zukunftsgerichteten Geschichtsphilosophie weiter auszuarbeiten, lohnt es sich, eine Brücke zur Ethik der Zukunft zu schlagen, die sich inzwischen als eigenständiger Diskurs etabliert hat. In ihm werden Fragen nach der moralischen Verantwortung für zeitlich nahe stehende oder entfernte Generationen gestellt. Es liegt auf der Hand, dass dabei Zeitverhältnisse eine wichtige Rolle spielen - nicht nur das Verhältnis von Vergangenheit, 
Gegenwart und Zukunft überhaupt, sondern auch speziell die ins Auge gefassten Zeiten der nahen oder fernen zukünftigen Generationen, für die Verantwortung übernommen werden soll.

Doch in den aktuellen Debatten über das Problem der Langzeitverantwortung wird das Thema Zeit und Geschichte wenig berücksichtigt. Demgegenüber beabsichtige ich zu zeigen, dass die Untersuchung der spezifisch temporalen und historischen Dimension der Verantwortung für zukünftige Generationen die ethische Argumentation zu ergänzen vermag. Für die Ethik der Zukunft bedeutet dies, dass sie sich gegenüber geschichtsphilosophischen Fragestellungen öffnet und bestimmte Einsichten in die Struktur und Funktion historischen Bewusstseins in ihre eigene Grundlegung integriert. Für die Philosophie der Geschichte hat das zur Konsequenz, dass sie sich mit der Zukunftsperspektive an der Lösung bestimmter Probleme der praktischen Philosophie beteiligt. Das Ziel besteht daher in einer Verbindung von Zukunftsethik und Geschichtsphilosophie.

An dieser Stelle knüpfe ich an meine vorausgegangen Überlegungen an, indem ich hier voraussetze, dass der zukünftigen Zeit als Wirkhorizont gegenwärtigen Handelns ein bestimmter Grad von Wahrscheinlichkeit zugeschrieben werden kann. Sofern man die Zukunft als Zeit-Raum von Handlungsmöglichkeiten fasst, lässt sie sich auch als spezifisch historische Zeit begreifen. Die Geschichtlichkeit der Zukunft resultiert letztlich aus individuellen und kollektiven Erwartungshaltungen sowie aus der Bewertung zukünftiger Güter und Interessen. Die Ethik der Zukunft hat es daher wesentlich mit einer zukünftigen Geschichte zu tun, deren Möglichkeit im Folgenden unterstellt wird.

Zunächst werde ich die mehr oder weniger impliziten Zeitstrukturen der Zukunftsethik kritisch analysieren. Dabei wird sich herausstellen, dass einige dieser Ethiken die Zeit teilweise ausblenden. Wenn einige Philosophen beklagen, die Ethik leide an Zukunftsvergessenheit, so bescheinige ich der gegenwärtigen Zukunftsethik eine tendenzielle Zeitvergessenheit. Über diese Kritik hinaus versuche ich in systematischer Perspektive eine geschichtsphilosophische Fundierung der Langzeitverantwortung, die sich auf den Standpunkt Gegenwart und auf die Geschichtlichkeit der Fernverpflichtung bezieht; sodann skizziere ich konkrete Modelle, die ich dafür geeignet halte, eine Verbindung zwischen den nahen und fernen Zeiten der Verantwortung zu konstruieren. 


\section{Ethische Geschichtsphilosophie und Historisierung der Ethik}

Wie zuvor meine ich mit dem Begriff »Geschichtsphilosophie« nicht diesen Denktyp in toto, sondern die philosophische Reflexion über historische Aspekte der Ethik. Das schließt nicht aus, dass bestimmte Elemente der `klassischen` Geschichtsphilosophie aktualisiert werden können, so wie auch Denkmotive des Historismus und anderer Strömungen zum Zuge kommen. In einem solchen Rückblick zeigt sich indessen, dass Geschichtsphilosophie und Ethik keineswegs immer so getrennt auftraten, wie es in der heutigen Ethik der Fall ist. Seit der Epoche der europäischen Aufklärung verschränkten sich beide Disziplinen miteinander, indem die Geschichtsphilosophie Ansätze einer Zukunftsethik enthielt und die Ethik infolge der Entstehung des historischen Denkens verzeitlicht wurde. In diesem Sinn kann man sowohl von einer ethischen Geschichtsphilosophie als auch von einer Historisierung der Ethik sprechen. An diesen Zusammenhang, der im vorliegenden Buch mehrfach angeklungen ist, möchte ich mit meinem Vorschlag einer Synthese von Ethik und Geschichtsphilosophie anknüpfen.

Auf der einen Seite stellt die Geschichtsphilosophie der Aufklärung eine frühe Form von Zukunftsethik dar. Das ergab sich das aus der utopischen Perspektive. Weil eine solche Ethik historisches Bewusstsein darüber voraussetzt, dass sich Vergangenheit, Gegenwart und Zukunft voneinander unterscheiden und dass die Geschichte einem steten Wandel unterworfen ist, verwundert es nicht, dass die Frage nach dem Beitrag der gegenwärtig lebenden Menschen für das Wohlergehen zukünftiger Generationen zuerst im Kontext der Geschichtsphilosophie gestellt wurde. Insbesondere die Fortschrittsidee drückte die Erwartung aus, dass sich die Lebensbedingungen der Menschen kontinuierlich verbesserten, so dass die zukünftige Zivilisation den gegenwärtig erreichten Zustand überträfe.

Unbegründet ist freilich der Vorwurf, die Geschichtsphilosophen forderten von den gegenwärtig lebenden Menschen den Verzicht auf ihre Glücksansprüche zugunsten zukünftiger Generationen. Bezeichnenderweise lässt sich diese Forderung in der von mir dargestellten französischen Geschichtsphilosophie des 18. Jahrhunderts nicht nachweisen. Es war vielmehr Kant, der das darin steckende moralische Problem auf ambivalente Weise explizierte. Zum einen mahnte er an die Pflicht der Menschen, die 
»Hoffnung besserer Zeiten« nicht aufzugeben, um den Willen zur praktischen Anstrengung wach zu halten. ${ }^{1}$ Zum andern kritisierte er die »befremdende« Vorstellung, »dass die ältern Generationen nur scheinen um der späteren willen ihr mühseliges Geschäft zu treiben, [...] ohne doch selbst an dem Glück, das sie vorbereiteten, Anteil nehmen zu können «. ${ }^{2}$ Diese Kritik am Fortschrittsglauben ist im Laufe der Zeit häufig geübt worden; bereits im 19. Jahrhundert war sie im deutschen Historismus verbreitet; im 20. Jahrhundert richtete sie sich vor allem gegen den Marxismus; sie hat sich bis in die gegenwärtige Ethik der Zukunft erhalten. ${ }^{3}$ Als Zielscheibe diente wiederum die Geschichtsphilosophie Hegels, der den Glücksanspruch der Individuen in der Weltgeschichte für zweitrangig erklärte. Während Volney angesichts des historisch erfahrbaren Unglücks in "Melancholie» fiel, hielt Hegel diese »Trauer« für unbegründet, weil sie den geschichtlichen Endzweck verkenne, »dem alle Opfer auf dem weiten Altar der Erde und in dem Verlauf der langen Zeit gebracht worden « sind. ${ }^{4}$ Es ist mir wichtig daran zu erinnern, dass in der Geschichtsphilosophie der Aufklärung weder die Forderung eines Glücksverzichts erhoben wurde noch die Distanzierung von einer derartigen Zumutung unausgesprochen blieb.

Auf der anderen Seite folgte auf das Geschichtsdenken eine Historisierung der Ethik - allerdings mit zeitlicher Verzögerung. Obwohl Hume in seinen Prinzipien der Moral Beispiele aus der Geschichte wählte und selbst Historiker war, konzipierte er die moralischen Maßstäbe noch völlig ungeschichtlich. Und obwohl Kant einige Schriften zur Philosophie der Geschichte verfasste, konstruierte er das Sittengesetz fern von der Vorstellung eines geschichtlichen Wandels. Erst im Utilitarismus des 19. Jahrhunderts machte sich das neue historische Bewusstsein bemerkbar. Indem Bentham und Mill den Folgen menschlichen Handelns besondere Beachtung

\footnotetext{
1 KANT, I. Zum ewigen Frieden, in: Werke in 12 Bänden, hg. von Wilhelm Weischedel, Frankfurt a. M. 1965, Bd. XI, 351.

2 KANT, I. Idee zu einer allgemeinen Geschichte in weltbürgerlicher Absicht, in: Werke, Bd. XI, 37.

3 Das kann hier nur exemplarisch belegt werden, etwa Heinrich Heine: Aufsatz über die verschiedene Geschichtsauffassung, in: Werke, Berlin 1972, Bd. 8, 233 f.; Leopold von Ranke: Über die Epochen der neueren Geschichte, hg. von Theodor Schieder und Hans Bending, München, Wien 1971, 54 f.; Hans Jonas: Das Prinzip Verantwortung. Versuch einer Ethik für die technologische Zivilisation, Frankfurt a.M. 1984 (1979), $45 \mathrm{f}$.

${ }^{4}$ Zu Volney siehe das zweite Kapitel; Georg Wilhelm Friedrich Hegel: Vorlesungen über die Philosophie der Geschichte, in: Werke in 20 Bänden, hg. von Eva Moldenhauer und Karl Markus Michel, Frankfurt a.M. 1969, Bd. 12, 33; zur Anspielung Hegels auf Volney vgl. Jacques d'Hondt: Hegel secret: recherches sur les sources cachées de la pensée de Hegel, Paris 1968, 83 ff.
} 
schenkten, erhielt die Ethik eine zeitliche Perspektive. ${ }^{5}$ Weil sie dabei - in der Tradition der Aufklärung - eine Verbesserung der Lebensverhältnisse anstrebten, machten sie die Ethik zu einem Unternehmen für die Zukunft. Wenn sie sich das »größte Glück der größten Zahl« zum Ziel setzten, schlossen sie das Glück der zukünftig lebenden Menschen ein. So wie die Glücksansprüche in der Gegenwart bilanziert wurden, legten sie die theoretische Basis für eine Bilanzierung des zu verteilenden Glücks von Gegenwart und Zukunft. An diese Art Zukunftsethik konnten die späteren Theoretiker der Langzeitverantwortung unmittelbar anschließen.

Den Auftakt zu einer expliziten Ethik der Zukunft gab indessen Jonas mit Das Prinzip Verantwortung, das sich in erster Linie auf die Verantwortung für zukünftige Generationen bezog. ${ }^{6}$ Er verzeitlichte die Ethik, indem er den Zeitraum der moralischen Verantwortung radikal in die Zukunft verlängerte. Den Grund sah er in einer neuen Größenordnung der "Fernwirkung" moderner Technologien, die der entsprechenden Verantwortung eine größere »Reichweite in die Zukunft« abverlangten. Auf Jonas berufen sich gegenwärtige Theoretiker der Langzeitverantwortung wie etwa Birnbacher in Verantwortung für zukünftige Generationen. Weil die technische Verfügungsmacht in die weite Zukunft reicht und die Gattungsexistenz der Menschheit aufs Spiel setzt, bedarf es der neuen Disziplin der Zukunftsethik, welche in der Lage ist, den Zeithorizont zu erweitern. ${ }^{7}$ Die theoretischen Mittel, um die Probleme der Langzeitverantwortung zu lösen, gewinnt Birnbacher aus der utilitaristischen Tradition, die schon Ansätze zu einer Zukunftsethik enthielt. Gemäß dem Utilitarismus soll die Lösung in einer Gesamtbilanz der Interessen der gegenwärtigen und zukünftigen Generationen bestehen.

Seit einigen Jahren werden die Chancen einer praktisch wirksamen Zukunftsethik jedoch wesentlich skeptischer beurteilt. Den Grund sieht man darin, dass der raum-zeitliche Horizont überschritten wird und damit der neue Typ der Verantwortung ins Ungewisse, Anonyme und Unverbindliche

\footnotetext{
5 Thomas Macho: Künftige Generationen. Zur Futurisierung der Ethik in der Moderne, in: Claus Langbehn (Hg.): Recht, Gerechtigkeit und Freiheit, Paderborn 2006, $318 \mathrm{ff}$.

6 JONAS, H. Das Prinzip Verantwortung, 9, 39, 64, 84, 220. - Zum Unterschied zwischen den Begriffen »Pflicht« und »Verantwortung« siehe Ludger Heidbrink: Grenzen der Verpflichtung. Zum Verhältnis von Verantwortung und Pflichten, in: Recht, Gerechtigkeit und Freiheit, a.a.O. (Anm. 5), 239-268.

7 BIRNBACHER, D. Verantwortung für zukünftige Generationen, Stuttgart 1988, $11 \mathrm{ff}$.
} 
zu laufen droht. ${ }^{8}$ Wenn man von den real erlebbaren Beziehungen zwischen den Generationen ausgeht, erscheint die Verantwortung für zeitlich entfernt lebende Menschen als Zumutung. ${ }^{9}$ Setzt man nämlich Generationen statt interessengeleitete Personen voraus, entsteht die Gefahr moralischer Überforderung. ${ }^{10}$ Schwierigkeiten bereiten auch die Bestimmung eines Adressaten der Fernverpflichtung und die Begründung der Motivation handelnder Subjekte. ${ }^{11}$

Diese Probleme sollen nun diskutiert werden, indem sie in den theoretischen Kontext von Zeitlichkeit und Geschichtlichkeit gestellt werden. Auf diese Weise möchte ich die anfängliche These stützen, dass die Reflexion auf die historische Dimension der Zukunftsethik zur Lösung der gesamten Problematik etwas beizutragen imstande ist. Damit stellen sich zwei Aufgaben: Zunächst gilt es, die temporalen und historischen Implikationen der vorliegenden Zukunftsethiken freizulegen. Darüber hinaus ist die geschichtsphilosophische Fundierung einer Ethik der Zukunft systematisch auszuarbeiten. Ich beginne mit dem kritischen Geschäft, indem ich in Fortsetzung des siebten Kapitels die in den Zukunftsethiken enthaltenen Zeitstrukturen expliziere und analysiere.

\section{Zeitstrukturen in ethischen Diskursen}

Auf einer elementaren Ebene operieren die Theoretiker der Langzeitverantwortung mit der Unterscheidung zwischen Vergangenheit, Gegenwart und Zukunft, wobei die Perspektive auf die Zukunft als das

8 GETHMANN, C. F. Langzeitverantwortung als ethisches Problem im Umweltstaat, in: Langzeitverantwortung im Umweltstaat, Bonn 1993, 7; Christoph Hubig: Langzeitverantwortung im Lichte provisorischer Moral, in: Jürgen Mittelstraß (Hg.): Die Zukunft des Wissens, Berlin 2000, 298 ff., insbes. 302.

9 LEIST, A. Ökologische Gerechtigkeit: Global, intergenerationell und humanökologisch, in: Julian NidaRümelin (Hg.): Angewandte Ethik. Die Bereichsethiken und ihre theoretische Fundierung. Ein Handbuch, Stuttgart 2005, $455 \mathrm{f}$.

10 STURMA, D. Die Gegenwart der Langzeitverantwortung, in: Recht, Gerechtigkeit und Freiheit, a.a.O. (Anm. 5), $221 \mathrm{ff}$.

11 GETHMANN, C. F. Wer ist der Adressat der Langzeitverantwortung? In: Carl Friedrich Gethmann und Jürgen Mittelstraß (Hgg.): Langzeitverantwortung. Ethik, Technik, Ökologie, Darmstadt 2008, 16 ff.; Dieter Birnbacher: Langzeitverantwortung - das Problem der Motivation, in: Gethmann / Mittelstraß (Hgg.), ebd., $29 \mathrm{ff}$. 
wesentlich Neue der gegenwärtigen Ethik angesehen wird. ${ }^{12}$ Diese Aussage ist nicht so trivial, wie sie erscheint, weil dagegen eingewendet werden kann, die Ethik sei immer schon auf Zukunft ausgerichtet. Jede moralische Regel und jeder Imperativ bezieht sich auf zukünftiges Handeln. Sofern dabei universelle Geltung beansprucht wird, ist in deren Bereich die Zukunft aus prinzipiellen Gründen eingeschlossen. Daher lässt sich behaupten, die Zukunft sei in der Ethik per definitionem enthalten, so dass es keiner "neuen Disziplin bedürfe. $^{13}$

An dieser Argumentation ist erkennbar, dass die Konzeption einer Zukunftsethik nur dann sinnvoll ist, wenn der entsprechende Zeitraum näher definiert wird. Wie sich bei den in die Zukunft projizierten »Fristen« zeigte, zielt auch die Langzeitverantwortung auf den Unterschied zwischen einer »kurzen« Zeit, der sich die klassische Ethik widmet, und einer »langen« Zeit, die den weiter reichenden Folgen moderner Technologien geschuldet ist. Während das »Ende« des eröffneten Zeithorizonts notgedrungen unbestimmt bleibt, weil sich die Wirkungen gegenwärtigen Handelns irgendwann verlieren, lässt sich hingegen die Zäsur zwischen "naher" und »ferner" Zukunft ziemlich präzise festlegen. Konsens besteht über die ethisch relevante Grenze nach jeweils drei Generationen, die gleichzeitig miteinander zusammen leben. ${ }^{14}$ Die Zeitspanne der "nahen« Generationen von Eltern, Kindern und Kindeskindern bzw. Großeltern und Enkeln entspricht den Anforderungen einer anwendungsorientierten Ethik, weil sie in ihrer Selbstverständlichkeit zur alltäglichen Praxis gehört. Besonders für Phänomenologen und Kommunitaristen ist diese Grenze so existentiell, dass sie jede Langzeitverantwortung nur auf der Basis einer solchen Nahverantwortung für möglich halten. ${ }^{15}$

Der Drei-Generationen-Rhythmus erfüllt bestimmte Kriterien, die diesem Zeitraum philosophische Bedeutung verleihen. Sie ist

\footnotetext{
12 JONAS, H. Das Prinzip Verantwortung, 27 f., 64; BIRNBACHER, Verantwortung für zukünftige Generationen, s. 15; Hubig, a.a.O. (Anm. 8), 296 f.; Leist, a.a.O. (Anm. 9), 453; Sturma, a.a.O. (Anm. 10), 227.

${ }^{13}$ So argumentiert Birnbacher, Verantwortung für zukünftige Generationen, 92-98; vgl. Gethmann, a.a.O. (Anm. 8), 4; ähnlich auf dem Gebiet des Rechts Johannes Caspar: Generationen-Gerechtigkeit und moderner Rechtsstaat. Eine Analyse rechtlicher Beziehungen innerhalb der Zeit, in: Dieter Birnbacher und Gerd Brudermüller (Hg.), Zukunftsverantwortung und Generationensolidarität, Würzburg 2001, 74. 14 JONAS, Das Prinzip Verantwortung, 215; BIRNBACHER, Verantwortung für zukünttige Generationen, 24 f., 156; Caspar, a.a.O. (Anm. 13), 82.

${ }^{15}$ GETHMANN, a.a.O. (Anm. 8), 12; Leist, a.a.O. (Anm. 9), 453 f.
} 
anthropologisch begründet, weil sie den Radius kognitiv erfahrbarer und emotional bewältigbarer Verantwortung begrenzt; sie ist sozialphilosophisch begründet, weil sie den Horizont der konkreten Lebenswelt in Familie und Gemeinschaft beschreibt; sie ist ontologisch begründet, weil es sich um Menschen handelt, die schon existieren und denen daher reale Interessen, Rechte und Pflichten zugeschrieben werden können. Da diese Kriterien für die darauf folgenden Generationen nicht mehr zutreffen, stellt sich die Frage, ob und wie die lebensweltlich legitimierte Verantwortung für die "nahen« Generationen auf die »fernen" Generationen ausgeweitet werden kann. In unserem Zusammenhang interessieren wiederum die in einer solchen Argumentation enthaltenen Zeitstrukturen.

Bereits Jonas reflektiert ansatzweise die "Zeitlichkeit« seiner Ethik, indem er die Gegenwart nicht nur in eine ethisch bedeutsame Zukunft verlängert, sondern auch innerhalb des "Horizonts der Zukunft" zwischen "Nah- und Fernhorizont " unterscheidet. ${ }^{16}$ Dabei erhebt er den Anspruch, die moralische Verantwortung aus der Nähe in die Ferne und damit auf die gesamte »künftige Menschheit« zu übertragen. Er postuliert eine »totale Verantwortung «, die für Menschen aller Zeiten in gleichem Maße gelten soll. Aus dieser Generalisierung zieht er die zeittheoretisch paradoxe Schlussfolgerung einer »fiktiven Gleichzeitigkeit mit den später Lebenden«. Auf diese Weise schlägt die "Zukunftsverantwortung" in eine "Gleichzeitigkeitsethik« um. Letztlich werden damit Zeit und Zeitlichkeit wieder zurückgenommen.

Eine ähnliche Paradoxie lässt sich in utilitaristischen Ethiken beobachten, deren Universalismus auf eine Tilgung der Zeitdifferenz hinausläuft. Das ergibt sich aus einer "Zukunftsbewertung", in der zukünftigen Interessen und Gütern derselbe Wert zugeschrieben werden soll wie denen der Gegenwart. Diese Position richtet sich gegen die so genannte Diskontierung, mit deren Hilfe Ökonomen Güter abwerten, die erst später zur Verfügung stehen. ${ }^{17}$ Während dieses Verfahren für produzierte Güter durchaus legitim ist, weil in Zukunft mit geringeren Kosten und niedrigeren Preisen zu rechnen ist, lässt es sich auf natürliche Güter und elementare

\footnotetext{
16 JONAS, H. Das Prinzip Verantwortung, 198 f., 215; zum Folgenden 89-91, 245.

17 BIRNBACHER, D. Läßt sich die Diskontierung der Zukunft rechtfertigen? in: Zukunftsverantwortung und Generationensolidarität, a.a.O. (Anm. 13), 117 ff.; Carl Friedrich Gethmann und Georg Kamp: Gradierung und Diskontierung bei der Langzeitverpflichtung, ebd., 147 f.; Dieter Cansier: Langzeitverantwortung und Diskontierung, in: Gethmann / Mittelstraß (Hgg.); a.a.O. (Anm. 11), 58 ff.
} 
Lebensbedingungen wie Luft und Wasser, die in der Umweltethik die entscheidende Rolle spielen, nicht mehr übertragen. Ebenso wenig sind die immer knapper werdenden Ressourcen wie etwa Öl und Gas einer Diskontrate unterworfen. Völlig unakzeptabel ist es schließlich, die Bedürfnisse und Interessen zukünftiger Menschen zu »diskontieren«, d.h. sie geringer zu bewerten als die Interessen der gegenwärtig Lebenden. Dagegen wehren sich die Ethiker zu Recht mit dem Argument, dass eine solche unterschiedliche Bewertung moralisch unhaltbar ist.

Wie plausibel dieser Einwand auch sein mag, so problematisch ist doch die zeittheoretische Konsequenz. Denn die berechtigte Kritik an der Abwertung der Zukunft führt dazu, allen Zeiten gleichen Wert zuzuschreiben. Aus der Feststellung, dass alle Menschen gleiche Rechte haben, unabhängig davon, in welcher Zeit sie leben, soll eine Gleichwertigkeit aller Zeiten folgen. Was bei Jonas "Gleichzeitigkeitsethik" hieß, taucht im Utilitarismus als temporale Gleichstellung wieder auf. An die Stelle der realen Gleichzeitigkeit der drei Generationen tritt die hypothetische Gleichzeitigkeit aller denkbaren Generationen. Jede Art »Zeitpräferenz« steht unter dem Verdacht, damit nur die gegenwärtigen Interessen verteidigen $\mathrm{zu}$ wollen. ${ }^{18}$ Wie der Ethiker in der Gegenwart einen unparteilichen Standpunkt einzunehmen beansprucht, so wird auch im Verhältnis der Zeiten eine "chronologische Unparteilichkeit" gefordert. Die ethische Position zeichnet sich gerade dadurch aus, dass sie von der konkreten Zeit absieht; universalistische Ethik bedeutet daher Abstraktion von bestimmten Zeitstrukturen. Zugespitzt formuliert: Diese Art Zukunftsethik ist Ethik ohne Zeit und damit letztlich auch ohne zukünftige Zeit.

\section{Vom Standpunkt der Gegenwart}

Nachdem ich die expliziten Äußerungen und impliziten Voraussetzungen der Zukunftsethiken hinsichtlich ihrer temporalen Strukturen kritisch beleuchtet habe, gelange ich nun zur systematischen Begründung. Wie angekündigt, geht es mir dabei um eine geschichtsphilosophische Fundierung der Langzeitverantwortung. Um eine

\footnotetext{
${ }^{18}$ BIRNBACHER, D. Verantwortung für zukünftige Generationen, 29, 35; mit Berufung auf Hare ders., a.a.O. (Anm. 13), 124; kritisch zu Birnbacher siehe Gethmann, Kamp, a.a.O. (Anm. 17), 147; vgl. Leist, a.a.O. (Anm. 9), $465 \mathrm{f}$.
} 
solche Verbindung von Ethik und Geschichtsphilosophie zu versuchen, verfahre ich im Folgenden zweistufig. Bevor ich konkrete Modelle zur Vermittlung von naher und ferner Verantwortung nenne, versuche ich eine allgemeine Grundlegung, die sich wiederum auf zwei Prinzipien stützt: in diesem Abschnitt auf das Prinzip der Unhintergehbarkeit der Gegenwart und im folgenden Abschnitt auf das Prinzip der Allgegenwärtigkeit des historischen Wandels.

Das Problem einer »zeitlosen« Langzeitverantwortung besteht darin, dass die Gegenwart übergangen wird und folglich die Verantwortung für zukünftige Generationen gleichsam direkt in die Zukunft springt. An die Stelle des kritisierten Präsentismus, dem man vorwirft, die Interessen der gegenwärtig Lebenden auf Kosten der Zukünftigen zu bevorzugen, tritt ein naiver Futurismus, der dazu auffordert, sich das Schicksal der fernen Zukünftigen zu Herzen zu nehmen und die später lebenden Menschen wie unsere Nächsten zu betrachten. Während der Präsentismus die Zukunft vernachlässigt, behandelt der Futurismus die Zukunft wie eine unmittelbare Gegenwart.

Um dieses Problem zu lösen, knüpfe ich noch einmal an Jonas an, der sich explizit mit der Geschichtsphilosophie auseinandersetzt. Er kritisiert das utopische Denken der Aufklärung bis Marx und Bloch, das die Gegenwart angeblich nur als »Vorgeschichte Menschen um der Zukunft willen zu opfern und damit die Gegenwart als Mittel für zukünftige Zwecke zu missbrauchen. ${ }^{19}$ Mit dieser Kritik spielt er nicht nur auf Kants Einwand an, sondern beruft sich auch ausdrücklich auf den Historismus von Ranke, indem er jeder Gegenwart einen Selbstzweck und damit einen eigenen Wert zuschreibt. Natürlich beabsichtigt Jonas mit dieser widersprüchlichen Argumentation nicht, die Gegenwart auf Kosten der Zukunft zu verabsolutieren; offenbar will er nur an die Stelle des Fortschrittsglaubens eine Ethik des Bewahrens gegenwärtig erreichter Standards setzen. Doch die tiefere Einsicht besteht darin, dass die Probleme der Zukunft allein vom Standpunkt der Gegenwart gelöst werden können.

\footnotetext{
19 JONAS, H. Das Prinzip Verantwortung, 86, 386-388, 393; vgl. Birnbacher, Verantwortung für zukünftige Generationen, 11; Dieter Birnbacher und Christian Schicha: Vorsorge statt Nachhaltigkeit Ethische Grundlagen der Zukunftsverantwortung, in: Zukunftsverantwortung und Generationensolidarität, a.a.O. (Anm. 13), 19 f., 24; Sturma, a.a.O. (Anm. 10), 221; Friedrich Rapp: Verantwortung im Kontext, in: Carmen Kaminsky und Oliver Hallich (Hg.): Verantwortung für die Zukunft, Berlin 2006, 28 f.; Wilhelm Vossenkuhl: Heute und morgen gut? Über den zeitlichen Sinn des Guten, ebd., 49.
} 
Die Gegenwart der Fernverpflichtung wird auch dann zum Programm, wenn man von den gegenwärtig handelnden Personen ausgeht, um ein Auseinandertreten von personalen Interessen und Langzeitverantwortung zu vermeiden. Vordergründig bricht hier der skizzierte Interessenkonflikt wieder auf, wenn es heißt, »der Augenblick ist der temporale Ort der Erfüllung von Selbstinteresse «. ${ }^{20}$ Aber der geschichtsphilosophisch bedeutsame Ertrag liegt darin, dass die Verantwortung an die gegenwärtig lebenden Personen geknüpft wird und dass allein aus dieser Perspektive der Gegenwart die in die Zukunft reichende Verantwortung abgeleitet werden kann. Auch wenn sich diese Analyse von "Zeitverhältnissen" nicht geschichtsphilosophisch ausgibt, interpretiere ich sie als eine philosophische Reflexion auf die historische Dimension der Zukunftsethik.

Was ich im Anschluss an McTaggarts Zeitontologie und Kosellecks Geschichtstheorie als Projekt einer Zukunft der Gegenwart formuliert habe, gilt hier für die Zukunftsethik, die ebenfalls von der Gegenwart auszugehen hat. Dabei lässt sich eine grundlegende Erkenntnis des Historismus zum Umgang mit der Vergangenheit auf den Erwartungshorizont der Zukunft übertragen. Droysen machte darauf aufmerksam, dass der Historiker die Vergangenheit nicht unmittelbar zum Gegenstand seiner Forschungen und Darstellungen hat, sondern nur indirekt mittels Zeugnisse und Dokumente, die zwar aus der Vergangenheit stammen, aber in der Gegenwart vorliegen. Ebenso steht die Darstellung auf dem Standpunkt der Gegenwart, aus deren Perspektive der Vergangenheit ein Sinn zugeschrieben wird. ${ }^{21}$ Diese Einsicht ist nun zur Lösung von Zukunftsproblemen nutzbar zu machen. Denn auf vergleichbare Weise erschließt sich die Zukunft nur vom Standpunkt der jeweiligen Gegenwart. Wenn dabei die Gegenwart sozusagen von der vergangenen Zukunft des Historikers zur gegenwärtigen Zukunft des Ethikers wandert, zeigen sich einige Analogien.

Wie es für den Historiker eine vergangene Zukunft gibt, die für Menschen, die in einer vergangenen Gegenwart lebten, Zukunft bedeutete, so stellt sich für den Ethiker die Zukunft immer als gegenwärtige Zukunft dar. Wie der Historiker es nicht unvermittelt mit vergangenen Ereignissen zu tun

\footnotetext{
${ }^{20}$ STURMA, D. a.a.O. (Anm. 10), 221 ff.; vgl. CASPAR, a.a.O. (Anm. 13), 100.

${ }^{21}$ DROYSEN, J. G. Historik, Textausgabe von Peter Leyh, Stuttgart-Bad Cannstatt 1977, 9; vgl. ebd., 422.
} 
hat, so versetzt sich der Ethiker nicht direkt in die Zukunft, indem er den zukünftig lebenden Menschen gleichwertige Interessen und Rechte unterstellt. Wie der Historiker die Vergangenheit aus den gegenwärtig vorhandenen Zeugnissen zu rekonstruieren hat, so muss der Ethiker sein Verhältnis zu den zukünftig lebenden Generationen erst noch konstruieren. Die Unhintergehbarkeit der Gegenwart bildet das Transzendental einer jeden Zukunftsethik.

Ich bezeichne diesen Gegenwartsbezug als transzendental, um zu verdeutlichen, dass es sich hier um ein Prinzip handelt, das jenseits der Auseinandersetzung um aktuelle Interessen Geltung beanspruchen kann. Denn die Identifizierung von Standpunkt der Gegenwart und Präferenz für gegenwärtige Interessen ist keineswegs zwingend, so wie die Abstraktion von der Gegenwart oder von der Zeit kein Garant für moralisch korrekte Entscheidungen sein muss. Daher ist die Reflexion auf die temporale Dimension ebenso unparteiisch wie der ethische Universalismus. Ist die Perspektive der Gegenwart einmal anerkannt, eröffnet sich überhaupt erst das Feld gegenwärtiger Debatten über die gerechte Verteilung zukünftiger Güter. Wenn die gegenwärtig lebenden Personen mit ihren kontrovers geführten Diskursen den Ausgang bilden, können überzogene Zumutungen und moralische Überforderungen leichter vermieden werden. Zugleich bietet die damit vorausgesetzte Interessenlage eine bessere Chance dafür, die Menschen zum praktischen Handeln zu motivieren. Primat der Gegenwart bedeutet, von der gegenwärtigen Lebenswelt ein möglichst realistisches Verhältnis zu den später lebenden Generationen aufzubauen.

\section{Die Geschichtlichkeit der Fernverantwortung}

Das zweite Prinzip meiner geschichtsphilosophischen Grundlegung der Zukunftsethik besteht darin, die Einzigartigkeit der Gegenwart als besondere historische Zeit anzuerkennen. Daraus folgt wiederum, dass auch mit der Andersartigkeit der Zukunft zu rechnen ist. Das Wissen darüber, dass sich Vergangenheit, Gegenwart und Zukunft voneinander unterscheiden, weil sie einem sozialen Wandel unterworfen sind, setzt ein historisches Bewusstsein voraus, das selbst Resultat einer geschichtlichen Entwicklung ist. Dies macht die Geschichtlichkeit der Langzeitverantwortung aus.

Wie im zweiten und siebten Kapitel dargestellt wurde, ist die Vorstellung einer historischen Veränderung, in der sich der 
Erwartungshorizont vom Erfahrungsraum bisheriger Geschichte abkoppelt, erst um die Mitte des 18. Jahrhunderts mit dem Beginn der Geschichtsphilosophie entstanden. Wenn sich die Geschichte nicht wiederholt und für die Zukunft etwas Neues erwarten lässt, können aus der Vergangenheit keine "Lehren" für zukünftiges Handeln gezogen werden. Diese Entdeckung wurde im Historismus des 19. Jahrhunderts noch radikalisiert, indem nicht nur kontinuierliche "Fortschritte auf wissenschaftlichen, technischen und wirtschaftlichen Gebieten angenommen wurden, sondern auch ein kultureller Wandel, der das Wertesystem von Gesellschaften erfasst. ${ }^{22}$ Demnach verbietet es sich, die aufeinander folgenden Epochen nach einem einheitlichen Maßstab zu beurteilen.

Überträgt man diese Erkenntnis auf die Zukunft, reicht es nicht aus, die gegenwärtige Entwicklung der wissenschaftlich-technischen Zivilisation in die Zukunft zu projizieren und dabei allenfalls prognostische Schwierigkeiten zuzugestehen. Für die ethische Urteilsfindung ist vielmehr der Umstand entscheidend, dass sich vor allem auch die Wertmaßstäbe der zukünftig Lebenden verändern werden. Was in der modernen Geschichtswissenschaft oder in der historischen Soziologie zum Standard gehört, erweist sich jedoch in der Zukunftsethik als nicht so selbstverständlich und findet dort erst in jüngster Zeit Eingang.

Utilitaristische Zukunftsethiken schreiben im Grunde die Fortschrittsidee der Aufklärung fort, indem sie unterstellen, dass zukünftige Generationen zwar neue und heute schwer vorhersehbare Technologien entwickeln, aber letztlich gleich bleibende Bedürfnisse und Interessen haben werden, so dass sich jederzeit eine Nutzensumme von Gütern und Glücksansprüchen berechnen lasse. Dagegen sind Zweifel angebracht mit dem Argument, dass die Idee des guten Lebens einen genuin zeitlichen Sinn hat und von historischen Brüchen eingeholt wird.23 Dieser Überzeugung schließen sich inzwischen auch Utilitaristen an, indem sie die prognostische Unsicherheit der Technikfolgeabschätzung nun auch durch die Ungewissheit über die in Zukunft veränderten Zielvorstellungen ergänzen. Demnach ist zu erwarten, dass sich auch die Kultur als "Wertegemeinschaft« fortentwickeln

\footnotetext{
22 Insbesondere Ernst Troeltsch: Der Historismus und seine Probleme. Erstes (einziges) Buch: Das logische Problem der Geschichtsphilosophie, 2. Neudruck der Ausgabe Tübingen 1922, Aalen 1977, 164 ff.

${ }^{23}$ Kritisch zu BIRNBACHER, VOSSENKUHL, a.a.O. (Anm. 19), 49.
} 
wird, die in späteren Zeiten jeweils neue Optionen ermöglicht. ${ }^{24}$ Wenn dabei von einer "offenen Zukunft" die Rede ist, drückt sich darin das seit dem Historismus standardisierte Geschichtsbewusstsein aus.

Es lässt sich sogar zeigen, dass in einer derart flexiblen Zukunftserwartung bestimmte Geschichtsbilder im Hintergrund wirksam sind. Denn fragt man, was Langzeitverantwortung konkret heißen mag, fließen mitunter Deutungsmuster über den generellen Verlauf der Geschichte ein. ${ }^{25}$ So stellt sich die grundsätzliche Frage, ob wir wollen, dass es zukünftigen Generationen besser gehen soll; oder ob wir es für ausreichend halten, dass angesichts drohender Klimakatastrophe und knapper Energieressourcen die erworbene Lebensqualität bestehen bleibt; oder ob wir notgedrungen in Kauf nehmen, dass sich die Lebensbedingungen langfristig verschlechtern, wenn sich der unvermeidbare Schaden nur in vertretbaren Grenzen hält.

Unschwer erkennt man in diesen Optionen die drei Verlaufsformen Fortschritt, Stagnation und Verfall. Die Fortschrittsidee schwingt im Utilitarismus mit, wenn eine Steigerung der Zivilisation angenommen und dann auch noch zugunsten zukünftiger Generationen zur Pflicht gemacht wird. Das Denkmuster der Stagnation schleicht sich ein, wenn etwa Jonas den Utopismus verurteilt und dazu aufruft, die gegenwärtige Natur und Kultur zumindest $\mathrm{zu}$ bewahren. Das Verfallsszenario kommt in aktuellen Zukunftsethiken ins Spiel, wenn offen darüber debattiert wird, ob eine Verschlechterung der Lebensbedingungen moralisch gerechtfertigt werden kann, wenn die natürlichen, technischen und organisatorischen Mittel erhalten bleiben, die es einer zukünftigen Generation ermöglichen, ihr kulturelles Niveau aus eigener Anstrengung wieder zu verbessern.

Doch ist in diesen Debatten ein gemeinsamer Grundsatz erkennbar, der sich dem geschärften Geschichtsbewusstsein verdankt. Wenn von der prinzipiellen Offenheit des Geschichtsverlaufs auszugehen ist, folgt daraus für die Zukunftsethik, dass nicht primär für bestimmte Güter vorgesorgt wird, weil man ja nicht wissen kann, ob diese von den zukünftigen Generationen geschätzt werden. Vielmehr ist es geboten, die Bedingungen der

\footnotetext{
${ }^{24}$ BIRNBACHER, SCHICHA, a.a.O. (Anm. 19), 19 f., 24; LEIST, a.a.O. (Anm. 9), 470 f.; STURMA, a.a.O. (Anm. 10), 230.

25 STURMA, a.a.O. (Anm. 10), 230; BIRNBACHER, SCHICHA, a.a.O. (Anm. 20), 24; HEUBACH, A.: Generationengerechtigkeit - Herausforderung für die zeitgenössische Ethik, Göttingen 2008, $42 \mathrm{f}$.
} 
Möglichkeit zu schaffen, dass verschiedenartige Güter zur Verfügung stehen. ${ }^{26}$ Ebenso wenig geht es um die Sorge für festgelegte Interessen später lebender Personen, sondern um die Entwicklung und Erhaltung von zumutbaren ökonomischen, sozialen und kulturellen Bedingungen für ein menschenwürdiges Leben. Ziel ist es, den Möglichkeitshorizont offen zu halten, indem Handlungsspielräume zur Verwirklichung je eigener Lebensentwürfe erhalten oder geschaffen werden.

In diesem Kontext mutiert die »Offenheit der Geschichte« zu einer ethischen Kategorie. Die historische Erfahrung, dass es in modernen Gesellschaften einen steten und beschleunigten Wandel gegeben hat, und die daraus ableitbare Erwartung, dass dieser Wandel auch in Zukunft eintreten wird, legt das praktische Verhalten nahe, diesen Wandel aktiv zu unterstützen. In diesem Licht mag der Utilitarismus »autoritär« anmuten, weil er das Glück der in Zukunft lebenden Menschen vorsorglich festschreibt. Wie so häufig droht die gut gemeinte Absicht in Bevormundung umzuschlagen.

Im Gegenzug folgt aus dem historischen Bewusstsein das moralische Gebot, den zukünftigen Generationen eine Veränderung ihrer Lebensweise nicht nur zuzugestehen, sondern auch durch gezielte Maßnahmen freizustellen. Die Ermöglichung des kulturellen Wandels wird zur moralischen Pflicht, die das Selbstbestimmungsrecht einer jeden Generation anerkennt. $^{27}$ So sollen wir beispielsweise nicht nur bestimmte Verkehrssysteme hinterlassen, sondern auch die Freiheit, bestimmte Systeme wieder rückgängig zu machen oder zu verändern wie überhaupt den Wert Mobilität neu zu definieren. Geschichtsphilosophisch formuliert, besteht die so ermöglichte Freiheit darin, generell den zivilisatorischen "Fortschritt» selbst zu bestimmen, indem auch zur Disposition steht, ob sich eine Generation auf einem bescheideneren Niveau andere Vorteile der Umwelt und sozialen Gerechtigkeit verschaffen kann.

Die Geschichtsbilder fungieren wie Hintergrundmetaphern, deren theoriebildender Einfluss nicht zu unterschätzen ist. Implizit bleiben auch die Bezugnahmen auf geschichtsphilosophische Positionen. Die Wende zum

\footnotetext{
${ }^{26}$ BIRNBACHER, SCHICHA, a.a.O. (Anm. 19), 19 f., 23; STURMA, a.a.O. (Anm. 10), 230; HUBIG, a.a.O. (Anm. 8), 309 f.; Leist, a.a.O. (Anm. 9), 471; Caspar, a.a.O. (Anm. 13), 100.

${ }_{27}$ Siehe die Beispiele zur Französischen Revolution bei Ohad Parnes, Ulrike Veder und Stefan Willer: Das Konzept der Generation. Eine Wissenschafts- und Kulturgeschichte, Frankfurt a.M. 2008, 97 f.
} 
Historismus kommt im Grundsatz der Gegenwart der Fernverantwortung zum Vorschein. Das gilt auch vom Prinzip der Offenheit, das ohne eine radikale Historisierung der Geschichte nicht denkbar ist. Doch von der klassischen Geschichtsphilosophie stammt die generelle Orientierung auf die Zukunft wie auch der ethische Anspruch. In diesem Sinn enthält eine reflexive Zukunftsethik eine historistisch ergänzte Geschichtsphilosophie oder einen um die Zukunftsperspektive erweiterten Historismus.

\section{Das historische Dilemma der Zukunftsethik}

Abschließend gelange ich zum zweiten Schritt meines systematischen Begründungsversuchs und nenne einige Modelle, die ich dafür geeignet halte, eine Verbindungslinie von der Gegenwart zur Zukunft zu ziehen. Um die Funktion zu präzisieren, ist es hilfreich, das spezifisch historische Dilemma der Langzeitverantwortung unter diesem Gesichtspunkt noch einmal zuzuspitzen. Denn es zeigte sich, dass die bisherigen Zukunftsethiken von der temporalen und historischen Dimension und damit von konkreten sozialen Beziehungen zwischen den Generationen zum großen Teil absehen. Um diese Abstraktion zu kompensieren, dienen fiktive Konstruktionen, die das Verhältnis der gegenwärtigen zu den zukünftig lebenden Menschen kalkulierbar machen sollen.

Die utilitaristische Variante besteht darin, bestimmte Interessen und Rechte der "fernen" Generationen zu unterstellen, die von den heute existierenden Personen »stellvertretend « wahrgenommen werden, um eine intergenerationelle »Nutzensumme« aufzustellen. ${ }^{28}$ Wie in der traditionellen Ethik die Interessen von Kindern und geistig Behinderten vertreten werden sollen, fungiert in der Zukunftsethik im Fall der noch nicht Geborenen das Modell der Stellvertretung. Die diskurstheoretische Variante konstruiert ihrerseits einen fiktiven Dialog zwischen den gegenwärtig lebenden und zukünftig zu erwartenden Menschen. So wird die Verbindung zur zukünftigen Menschheit durch "Antizipation einer unbefristeten Diskursgemeinschaft « hergestellt. ${ }^{29}$ Die größte Verbreitung hat in dieser Hinsicht der »Generationenvertrag« nach dem Vorbild von Rawls'

\footnotetext{
${ }^{28}$ BIRNBACHER, Verantwortung für zukünftige Generationen, 101 ff.; vgl. Klaus Kornwachs: Das Prinzip der Bedingungserhaltung. Eine ethische Studie, Münster 2000, $63 \mathrm{f}$.

${ }^{29}$ GETHMANN, a.a.O. (Anm. 8), 12.
} 
Gerechtigkeitstheorie. Die Metapher "Schleier des Unwissens" wird so modifiziert, dass sie das Unwissen über die zeitliche Abstammung der Beteiligten einschließt. In diesem Fall sehen wir uns mit der "gleichzeitigen Versammlung aller nur denkbaren Generationen « konfrontiert. ${ }^{30}$

Die Stärke derartiger Konstruktionen liegt zweifellos in ihrem universellen Geltungsanspruch, der es erlaubt, das Prinzip Verantwortung auf die entferntesten Generationen auszudehnen. Doch die Grenzen kommen in der mangelnden Motivation zum Ausdruck, wodurch sich die Fernpflicht zum schwachen Appell verflüchtigt.

Eine Alternative stellt der Kommunitarismus dar, deren Vertreter sowohl den abstrakten Utilitarismus als auch den hypothetischen Kontraktualismus kritisieren. Dagegen bieten sie die konkreten Beziehungen zwischen den drei miteinander lebenden Generationen auf, die bereits Elemente einer Zukunftsverantwortung enthalten. Eltern haben ein elementares Interesse an ihren Kindern, für die sie sorgen müssen. Das Prinzip einer solchen Ethik ist die familiäre Fürsorge. ${ }^{31}$ Der unbestrittene Vorteil dieser Verantwortung für die nahen Verwandten besteht, wie angedeutet, im praktisch erfahrenen, empfundenen und motivierenden Lebens-zusammenhang. Aber zugleich macht sich die zeitliche Grenze der drei Generationen bemerkbar, die auf der schmalen moralischen Basis der Elternsorge nicht überschritten werden kann. An diesem neuralgischen Punkt stellt sich erneut die Frage, wie die praktische Lebenswelt der nahen Zukünftigen auf die fernen Zukünftigen übertragen werden kann.

Im Rahmen des Konstruktivismus wird vorgeschlagen, die Verantwortung für die nahen Zukünftigen wenigstens zum Ausgangspunkt für die Begründung der Langzeitverantwortung zu wählen, um die Nahverpflichtung in die ferne Zukunft erweitern zu können. ${ }^{32}$ Zwar ist es ethisch nicht gerechtfertigt, zukünftige Güter und Interessen zu »diskontieren«, aber daraus folgt nicht, dass es zwischen Nah- und Fernpflicht überhaupt keinen Unterschied gibt. Es ist ja nicht gleichgültig, ob Eltern für ihre eigenen Kinder sorgen oder ob sie sich für das Wohl einer

\footnotetext{
${ }^{30}$ CASPAR, a.a.O. (Anm. 13), 96; siehe John Rawls: Eine Theorie der Gerechtigkeit, Frankfurt a.M. 1979, $319 \mathrm{ff}$.

31 JONAS, Das Prinzip Verantwortung, 197 f.; LEIST, a.a.O. (Anm. 9), 459 f.; CASPAR, a.a.O. (Anm. 13), $92 \mathrm{f}$.

32 GETHMANN, a.a.O. (Anm. 8), 11, 15; GETHMANN, KAMP, A.a.O. (Anm. 17), 145, 149; vgl. STURMA, a.a.O. (Anm. 10), 235; kritisch dazu Hubig, a.a.O. (Anm. 8), 300.
} 
beliebig späten Generation Sorgen machen sollen. Um diesen lebensweltlichen Unterschied abzubilden, ohne in die Falle der Diskontierung zu laufen, wird der Begriff der Graduierung aufgeboten, der den Universalismus vermeidet und zugleich an der Langzeitverantwortung festhält. Unter dieser Voraussetzung kommt es darauf an, die Fernpflicht aus der Nahpflicht zu konstruieren, indem man aus der Erfahrung mit der nahen Zukunft eine moralische Verhaltensweise für die ferne Zukunft ableitet. Ziel ist es, nach dem Modell der Fürsorge eine konkrete Beziehung zwischen den nahen und entfernt gedachten Generationen vorzustellen.

Das konstruktivistische Verfahren enthält jedoch einen Widerspruch, der darin besteht, dass ein Modell der Fürsorge zugrunde gelegt wird, das es ja gerade zu überwinden gilt. Wenn die Elternsorge auf drei Generationen begrenzt bleibt, ist es erforderlich, ein anderes Modell zu finden, das es erlaubt, trotz historischer Brüche Kontinuitäten zwischen den nahen und mittleren Zeiten der Verantwortung herzustellen. Gesucht wird nach einem Modell, das zwar nicht so lebensnah sein kann und darf wie das Sorgemodell, aber doch konkreter ist als die Denkmuster der unbefristeten Diskursgemeinschaft, der intergenerationellen Nutzenbilanzierung oder des generationenübergreifenden Vertrags. Zur Überwindung dieses Dilemmas schlage ein Modell vor, das aus dem Bereich der Geschichte stammt: das Modell der Erbschaft in der Generationenfolge.

\section{Modelle der Langzeitverantwortung: Generationenfolge und Erbschaft}

Um das Modell der Erbschaft einzuführen, erläutere ich zunächst das der Generationenfolge. Dazu ist der Begriff der Generation zu klären, der folgende Arten von Vermittlung leistet: zwischen 1. Natur und Kultur, 2. Individuum und Menschheit, 3. Synchronie und Diachronie und 4. zwischen kurzer und langer Dauer. Wenn man nicht bei der biologischen Vererbung stehen bleiben will, ist das Vermächtnis einer jeden Generation als kulturelles Erbe zu fassen. Nicht zuletzt soll das Modell der Erbschaft in der Generationenfolge zur Motivation der Langzeitverantwortung beitragen.

\section{Generationenfolge}

Überträgt man die Frage nach dem »Subjekt« der Geschichte auf das Gebiet der Zukunftsethik, stehen drei Kandidaten zur Verfügung: Gattung, Generation und Person. In den Anfängen der Zukunftsethik wurde die 
Verantwortung für die »künftige Menschheit« postuliert bzw. der Fortexistenz der menschlichen "Gattung « ein hoher Wert zugeschrieben, so wie deren befürchtete Vernichtung als eine zu verhindernde moralische Katastrophe galt. ${ }^{33}$ Von diesem Pathos ist in späteren Ethiken der Zukunft nicht mehr die Rede. Wie selbstverständlich spricht man stattdessen von "Generationen«, die als gleichzeitig lebende oder zeitlich aufeinander folgende Altersgruppen definiert werden. ${ }^{34}$ Dagegen richtet sich eine Kritik, die dem Generationenbegriff eine soziale und ethische Bedeutung pauschal aberkennt und an dessen Stelle den Begriff der Person als einziges Subjekt und Adressaten von Verantwortung setzt. ${ }^{35}$ Trotzdem halte ich es für sinnvoll, den Begriff der Generation zur Vermittlung von Nah- und Fernverantwortung zu verwenden, ohne damit ein neues Handlungssubjekt unterstellen zu wollen. Doch ist zu berücksichtigen, dass Personen in sozialen Zusammenhängen agieren und kollektiv verantwortlich gemacht werden können. Unter dieser Voraussetzung versuche ich, den Begriff der Generation in eine geschichtsphilosophische Kategorie mit den genannten vier Aspekten umzuformulieren.

Erstens vermittelt der Generationenbegriff, wie sich im vierten Kapitel zeigte, die beiden Seiten Natur und Kultur. ${ }^{36}$ Denn das Wort generatio bedeutet Zeugung im Sinne der natürlichen Reproduktion der Geschlechter. Setzt man für eine Generation dreißig Jahre an, beträgt für Erwachsene die Dauer der Nahpflicht ungefähr fünfzig Jahre - eine biologische bemessene Zeitspanne, die philosophisch noch unterbestimmt bleibt. Zugleich hat der Begriff der Generation eine kulturelle Bedeutung, wie die Generationenforschung der Geschichtswissenschaft belegt, in der beispielsweise die Kriegsgeneration, 68er-Generation usw. zu den aktuellen Themen gehören. ${ }^{37}$ In unserem Kontext knüpft sich daran die Erwartung, dass der dabei verwendete Kulturbegriff, wie vage er auch erscheinen mag, zur Vermittlung von familiärer Sorge und Langzeitverantwortung beitragen könnte.

\footnotetext{
33 JONAS, Das Prinzip Verantwortung, 86, 245; Birnbacher, Verantwortung für zukünftige Generationen, 102.

${ }^{34} \mathrm{HEUBACH}$, a.a.O. (Anm. 25), $28 \mathrm{ff}$.

${ }^{35}$ STURMA, a.a.O. (Anm. 10), 226.

${ }^{36}$ WEIGEL, S. Genea-Logik. Generation, Tradition und Evolution zwischen Kultur- und Naturwissenschaft, Paderborn 2006, 9, 109; Parnes u.a., Das Konzept der Generation, a.a.O. (Anm. 27), 11.

${ }_{37}$ JUREIT, U. Generationenforschung, Göttingen: Vandenhoeck \& Ruprecht 2006.
} 
Zweitens hat der Generationenbegriff den Vorzug, dass er zwischen den Ideen der Menschheit und des Individuums auf einer mittleren Ebene angesiedelt ist. Einerseits vermeidet er den Rückgriff auf das Großsubjekt menschliche Gattung, das in der Geschichtsphilosophie der Aufklärung bis Hegel maßgebend war und schon vom Historismus zu Recht verabschiedet wurde. Nachdem sich auch in der Zukunftsethik das Referenzsubjekt "Gattung" als überdimensioniert herausstellte, verspricht der Generationenbegriff überschaubare und vielfältige Populationen. Auch in diesem Fall handelt es sich um Referenzsubjekte, ${ }^{38}$ die dadurch definiert sind, dass ihnen historische Ereignisse widerfahren. Mit Blick auf die Zukunft ist hier zum Beispiel zu befürchten, dass bestimmte Umweltschäden das Leben abgegrenzter Generationen auf unterschiedliche Weise beeinträchtigen. Der Generationenbegriff kann hier eine kritische Funktion ausüben, weil er die vermeintlich gleichmäßige Betroffenheit »aller" Menschen in Frage stellt.

Die beiden Aspekte verweisen drittens auf die sowohl synchrone als auch diachrone Dimension des Generationenbegriffs. ${ }^{39}$ In der Soziologie ist dieser Begriff prominent geworden, indem er altersspezifische Gruppen beschreibt, die gemeinsame Einstellungen und Lebensstile teilen. ${ }^{40}$ Generation bedeutet hier eine Erlebnis- und Erfahrungsgemeinschaft in sozialer Gleichzeitigkeit. Gegenüber dieser soziologischen Dominanz tritt der historische Aspekt in den Hintergrund. ${ }^{41}$ Denn Generation bedeutet ebenso die zeitliche Abfolge sozialer Kollektive, die man als Kulturgeschichte verstehen kann. Das entsprechende Modell ist das kulturelle Erbe, ${ }^{42}$ das sich auch für die Zukunftsethik eignet.

Im Anschluss daran vermittelt der Generationenbegriff viertens zwischen der kurzen und langen Dauer der Geschichte. Die Kategorie der Generation garantiert nicht nur soziologisch begrenzte Akteure, sondern in

\footnotetext{
38 Zum Unterschied zwischen Handlungssubjekt und Referenzsubjekt in der Geschichte siehe Hermann Lübbe: Geschichtsbegriff und Geschichtsinteresse. Analytik und Pragmatik der Historie, Basel, Stuttgart 1977, 122 f.; vgl. Johannes Rohbeck: Technik - Kultur - Geschichte. Eine Rehabilitierung der Geschichtsphilosophie, Frankfurt a.M. 2000, 174.

${ }^{39}$ WEIGEL, Genea-Logik, a.a.O. (Anm. 36), 108; Parnes u.a., Das Konzept der Generation, a.a.O. (Anm. 27), $10 \mathrm{f}$;; in diesem Zusammenhang ist auch von einem temporalen und intertemporalen Generationenbegriff die Rede: Heubach, a.a.O. (Anm. 25), 29.

${ }^{40}$ MANNHEIM, K. Das Problem der Generation, in: ders.: Wissenssoziologie. Auswahl aus dem Werk, eingeleitet und herausgegeben von Kurt H. Wolf, Berlin, Neuwied: Luchterhand 1964, 509-565.

${ }^{41}$ DILTHEY, W. Über das Studium der Wissenschaft vom Menschen, der Gesellschaft und dem Staat, in: Gesammelte Schriften, Bd. 5, Stuttgfart 1964, 35 f.

${ }^{42}$ WEIGEL, Genea-Logik, a.a.O. (Anm. 36), 62.
} 
der Zeitenfolge auch historisch mittlere Sequenzen. Diese Dimensionierung entspricht den formulierten Grundsätzen, ausgehend von der Gegenwart in planbaren und vorhersehbaren Fristen zu agieren und darüber hinaus nach jeder Generation die Ziele jeweils neu bestimmen zu lassen. Wenn gefordert wird, vor allem die Bedingungen für alternative und frei wählbare Möglichkeiten zu schaffen, ist diese Chance einer Neubestimmung den nachfolgenden Generationen freizustellen. Das Recht zur demokratischen Selbstbestimmung einer jeden Generation setzt das historische Denken in Kategorien der biologisch und kulturell bestimmten Generation voraus. Auf diese Weise kann der Begriff der Generation dazu beitragen, zwischen Nahund Fernverantwortung zu vermitteln.

Die aufeinander folgenden Generationen sind auch als eine »Kette der Lebewesen« zu betrachten, die im 18. Jahrhundert, so das vierte Kapitel, historisiert und in die Zukunft projiziert wurde. Der Sinn bestand darin, ein Gefühl der Solidarität zwischen den Generationen zu erzeugen mit der Aufforderung, am Wohl zukünftig lebender Menschen mitzuwirken. Im Historismus wurde die Generationenkette in die Vergangenheit verlegt, um aus dem so gewonnenen Geschichtsbewusstsein Orientierung für die damalige Gegenwart zu schöpfen. Eine so gewonnene historische Identität lässt sich auch auf die »Kette» der zukünftigen Generationen übertragen. Wie wir dazu aufgerufen werden, eine historisch begründete Haltung zu Europa zu entwickeln, so scheint es sinnvoll zu sein, ein solches Identitätsbewusstsein auch bei Themen der Zukunftsethik zu fördern. Angesichts der Anonymität der Zukünftigen bedarf es einer Reflexion der eigenen historischen Position in der Kette der Generationen und eines generationen-übergreifenden Bewusstseins.

Freilich reicht es nicht aus, sozusagen auf den Selbstlauf dieser »Kette« $\mathrm{zu}$ setzen, indem man darauf vertraut, dass sich die unmittelbare Vorsorge der Eltern für ihre Kinder überträgt, die dann ihrerseits für ihre Kinder sorgen, so wie die Eltern für sie vorgesorgt haben. ${ }^{43}$ Das Kalkül soll hier darin bestehen, wenn die gegenwärtige Generation für die eigenen Kinder vorsorge, gehe es den Urenkeln möglicherweise besser als mit moralischen Appellen zur Verantwortung für fern stehende Generationen. Aber mit dieser Begründung wird die Zukunftsethik wieder auf das ursprüngliche Modell der Elternsorge reduziert, als ob das Problem der Erweiterung dieses Modells gar

\footnotetext{
${ }^{43} \mathrm{Im}$ Anschluss an Passmore so Birnbacher, Langzeitverantwortung, a.a.O. (Anm. 11), $32 \mathrm{f}$.
} 
nicht bestünde. Wie sehr es zu begrüßen ist, die Ethik der Zukunft auf ein lebensweltliches $\mathrm{Maß} \mathrm{zu}$ bringen, so bedarf es eines weiter führenden Modells, das die Langzeitverantwortung mittlerer Reichweite zu begründen hilft. Diese Anforderung erfüllt das Modell der kulturellen Erbschaft.

\section{Erbschaft}

Fragt man nach der Art der »Verkettung « zwischen den Generationen, ist über die biologische Linie hinaus die Generationenfolge als eine Tradierung von Kultur zu verstehen. Dazu halte ich den Begriff des kulturellen Erbes für geeignet. ${ }^{44}$ Das Modell der Erbschaft vermag die gesuchte Vermittlung zwischen den Generationen zu leisten, weil es die engen Grenzen der familiären Fürsorge überwindet und zugleich wesentlich konkreter ist als die Konstruktionen universalistischer Theorien. Außerdem vermittelt der Erbschaftsbegriff zwischen Natur und Kultur, indem er sowohl an die biologische Vererbung anschließt als auch und vor allem die kulturelle Überlieferung abdeckt. Er bezieht sich zunächst auf die juristisch geregelte Erbschaft materieller Vermögen, dann auch auf kulturelle Vermächtnisse. Im Kontext der Zukunftsethik lässt er sich auf die kulturell überformten natürlichen Lebensbedingen ausdehnen: Wir "vererben" auch Kulturlandschaften einschließlich ihrer natürlichen Ressourcen.

Erbe bedeutet zunächst Weitergabe durch den Erblasser im Sinne von Tradierung, zugleich Fortführung, Vermehrung oder Veränderung durch den Erben. Turgot betrachtet die Kenntnisse der Menschen als einen Schatz, »den eine Generation an die nächste weitergibt wie eine Erbschaft, die um die Entdeckungen jedes Jahrhunderts erweitert wird $« .{ }^{45}$ Indem das Erbe zwischen Erblasser und Erben eine eigene Realität bildet, ermöglicht es einen flexiblen Gebrauch. ${ }^{46}$ Es determiniert nicht seine zukünftige Verwendung, sondern stellt es dem Erben frei, wie er von ihm Gebrauch machen will. In diesem Sinn stellt das Erbe die Bedingungen von Handlungsmöglichkeit dar. Es ist

\footnotetext{
${ }_{44}$ Siehe die Ausführungen zum Begriff des kollektiven Erbes bei Lukas H. Meyer: Historische Gerechtigkeit, Berlin, New York 2005, $135 \mathrm{ff.}$

45 TURGOT, A. R. J. Über die Fortschritte des menschlichen Geistes, hg. von Johannes Rohbeck und Lieselotte Steinbrügge, Frankfurt a.M. 1990, 140.

${ }^{46}$ Zum flexiblen Gebrauch technischer Gegenstände und Systeme siehe ROHBECK, a.a.O. (Anm. 38), $118 \mathrm{ff}$.
} 
mit dem geforderten »offenen« Charakter des Geschichtsprozesses vereinbar, indem es das Selbstbestimmungsrecht einer jeden Generation respektiert.

Die Grundidee meines Vorschlags, den Begriff der Erbschaft zur Begründung der Langzeitverantwortung heranzuziehen, besteht darin, das Thema positiv zu besetzen und dadurch mehr Motivation zu schaffen. Wenn ständig an die Zeitgenossen appelliert wird, drohende Katastrophen zu verhindern und die Menschheit zu retten, mag das zwar gut gemeint sein, aber besonders motivierend wirken solche Imperative nicht. Setzt man stattdessen die Erbschaft als Leitidee ein, lässt sich das Motiv verstärken, dass die Menschen die Errungenschaften ihrer Kultur an die späteren Generationen überliefern wollen. Wer die Malerei eines Caspar David Friedrich schätzt, legt Wert darauf, dass dessen Gemälde gut konserviert werden und auch noch von zukünftigen Generationen betrachtet werden können. ${ }^{47}$ Das Modell der Erbschaft geht sogar über einen solchen Wunsch hinaus, weil es auch die Art und Weise der Tradierung zu strukturieren erlaubt. Dabei spielt nicht nur das Anliegen des Erblassers, sondern auch das antizipierte Verhalten des Erbenden eine Rolle. Auch in diesem Fall knüpft das Modell an die lebensweltliche Praxis des Vererbens an und weist zugleich über die drei Generationen hinaus.

Ferner kommt das anthropologisch fundierte Motiv ins Spiel, die eigne Person $\mathrm{zu}$ transzendieren und damit den individuellen Tod $\mathrm{zu}$ überschreiten, um im kulturellen Erbe virtuell fortzuleben. Die Menschen wehren sich gegen das Vergessenwerden und versuchen, sich mit Hilfe ihrer Erbschaft als eines symbolischen Kapitals das Gedenken zukünftiger Generationen zu sichern. ${ }^{48}$ Auf diese symbolische Weise scheint die Asymmetrie der Langzeitverantwortung überwindbar, da man sich nun auch von den später Lebenden eine Gegenleistung verspricht. Es entsteht das Bild eines wechselseitigen Verhältnisses von praktischer Fernpflicht und erwarteter Gedenkpflicht.

Die Erwartung des guten Gedenkens ist eine antizipierte Erinnerung, welche die zeitliche Struktur des Zweiten Futurs hat. Was im siebten Kapitel im Kontext "zukünftiger Vergangenheit« kritisch kommentiert wurde, soll an dieser Stelle konstruktiv gewendet werden. Nachdem zukünftige

\footnotetext{
47 BIRNBACHER, Langzeitverantwortung, a.a.O. (Anm. 11), 33 f..

${ }^{48}$ Zum Begriff des symbolischen Kapitals siehe Pierre Bourdieu: Die feinen Unterschiede, Frankfurt a.M. Suhrkamp 91997, $115 \mathrm{ff}$.
} 
Generationen bestimmte Folgen unseres gegenwärtigen Handelns zu spüren bekommen haben, werden wir dafür nicht nur verantwortlich sein, sondern sehr wahrscheinlich auch rückwirkend dafür verantwortlich gemacht werden. Demnach ist eine Person fernverantwortlich, weil sie sich in der Zukunft retrospektiv zu verantworten hat für Leistungen und Fehler, welche in der Gegenwart entstanden sind. Wer Verantwortung im zukunftsbezogenen Sinn übernimmt, erklärt sich bereit, zu einem zeitlich späteren Zeitpunkt Rechenschaft abzulegen und sich dafür zur Verantwortung im vergangenheitsorientierten Sinn ziehen $\mathrm{zu}$ lassen. ${ }^{49}$ Die prospektive Verantwortung wird also aus einer retrospektiven Sichtweise abgeleitet, die eine Antizipation der Zukunft voraussetzt. Ich bezeichne diese Zeitstruktur als prospektive Retrospektivität der Langzeitverantwortung

Daraus könnte der Wunsch hervorgehen, dass man nicht nur für sein Fehlverhalten zur Rechenschaft gezogen werden möchte, sondern das legitime Interesse hat, für eine gelungene Vorsorge in guter Erinnerung zu bleiben. Formuliert man diesen Zusammenhang in Kategorien eines in die Zukunft übertragenen Erinnerungsdiskurses, lässt sich von einer möglichen Schuld gegenüber den Opfern der Zukunft sprechen, so wie die zukünftig Lebenden uns Dank schulden, sofern wir hinsichtlich ihrer Lebensbedingungen nicht schuldig geworden sind. Das Modell des konkreten Erbes, das von einer Generation zur anderen weitergegeben wird, könnte dieser Denkfigur mehr Realismus verschaffen.

Mit der Denkfigur Erinnerung an die Zukunft verweise ich wieder auf die vorausgegangene zeit- und geschichtstheoretische Begründung der Langzeitverantwortung. Ich hoffe damit demonstriert zu haben, dass die Philosophie der Zukunft dazu fähig ist, die Zukunftsethik theoretisch anzureichern. Die wichtigsten Theoreme bestanden im Gegenwartsbezug der Fernverantwortung mit einer entsprechenden Begrenzung der Reichweite moralischer Verpflichtungen, in der Einsicht in die Geschichtlichkeit der Gegenwart und Zukunft sowie in der prospektiven Retrospektivität der Langzeitverantwortung. Das hatte zur praktischen Folge, dass die Beziehung zwischen gegenwärtigen und zukünftigen Generationen ansatzweise symmetrisch gedacht wird in der Weise, dass beim späteren Umgang mit der tradierten Kultur frei wählbare und alternative Optionen mitgedacht werden. Die Erbschaft in der Generationenfolge ist ein Modell dafür, dass nicht nur

${ }^{49}$ HUBIG, A.a.O. (Anm. 8), 297; BIRNBACHER, a.a.O. (Anm. 11), 23. 
das Deutungsmuster der familiären Vorsorge überschritten werden kann, ohne zu abstrakten Adressaten Zuflucht nehmen zu müssen, sondern dass auch die Vererbung kultureller Güter als kontingenter und wechselseitiger Handlungszusammenhang vorstellbar wird.

Wie die Ethik durch die geschichtsphilosophische Reflexion einen historischen Charakter erhält, so wird die Philosophie der Geschichte durch die Ethik ergänzt. Dabei hat sich gezeigt, dass sowohl die Zukunftsorientierung als auch die ethische Dimension zur Geschichtsphilosophie seit der Aufklärung gehören und dass es daher gute Gründe gibt, an die Philosophie dieser Epoche anzuknüpfen. Ziel ist es, die Geschichtsphilosophie aus dem Bann der geschichtstheoretischen Methodologie zu lösen und in den Kontext der praktischen Philosophie zu stellen.

E-mail: johannes.rohbeck@tu-dresden.de

Recebido: 01/2010

Apovado: 02/2010 